JOURNAL OF THE

AMERICAN MATHEMATICAL SOCIETY

Volume 17, Number 4, Pages 983-994

S 0894-0347(04)00460-6

Article electronically published on May 25, 2004

\title{
CYCLES REPRESENTING THE TODD CLASS OF A TORIC VARIETY
}

\author{
JAMES POMMERSHEIM AND HUGH THOMAS
}

\section{INTRODUCTION}

Shortly after toric varieties were introduced in the 1970's, Danilov Dan asked whether it is possible, given a lattice $N$, to assign a rational number $\mu(\sigma)$ to every cone $\sigma$ in $N$ so that for any fan $\Sigma$ in the lattice, the Todd class $\operatorname{Td}(X)$ of the corresponding toric variety $X$ would be given by

$$
\operatorname{Td}(X)=\sum_{\sigma \in \Sigma} \mu(\sigma)[V(\sigma)],
$$

where $[V(\sigma)]$ is the class in the Chow group of $X$ corresponding to the orbit closure $V(\sigma)$. Note that the above formula is to hold simultaneously for all fans in $N$. (The much weaker statement that the Todd class of any fixed toric variety can be written as some linear combination of the $[V(\sigma)]$ follows from the fact that the $[V(\sigma)]$ generate the Chow group of $X$.)

Morelli answered Danilov's question by showing (non-constructively) the existence of such an assignment [Mor, Theorem 3]. He also gave an explicit canonical construction of such an assignment, where the $\mu(\sigma)$ are rational functions on a certain Grassmannian [Mor, Theorem 11]. In this paper, we construct assignments satisfying Danilov's conditions, in which the coefficients $\mu(\sigma)$ are rational numbers (Corollary 1).

The present work also serves to unify two previous formulas for the Todd class of a toric variety: Morelli's canonical expression for the Todd class in terms of rational functions on Grassmannians [Mor Theorem 11] and the first author's expression for the Todd class of a simplicial toric variety as a polynomial in the torus-invariant divisors [Pom2]. In particular, we show that if this polynomial expression is multiplied out using the ring structure developed in Tho, we obtain Morelli's canonical formula (Corollary 2).

One motivation for Danilov's question is the connection between Todd classes of toric varieties and counting lattice points in polytopes. We will now briefly sketch the application of our results to this problem. If $P$ is a lattice polytope in $\mathbb{Z}^{n}$, and $F$ is a face of $P$, there is an associated outer normal cone, which we denote by $C(F, P)$. Also associated to $F$ is a lattice volume, $\operatorname{vol}(F)$ : this is the volume of $F$ normalized with respect to the lattice in the affine span of $F$. In this paper, we construct a map $\mu$ from rational cones in $\mathbb{Z}^{n}$ to $\mathbb{Q}$ with the following property:

Received by the editors October 25, 2003.

2000 Mathematics Subject Classification. Primary 14M25; Secondary 14C17, 52B20.

Key words and phrases. Toric variety, Todd class, polytopes, counting lattice points.

(C)2004 American Mathematical Society

Reverts to public domain 28 years from publication 
for any lattice polytope $P$ in $\mathbb{Z}^{n}$, the number of lattice points in $P$ is given by the formula

$$
\#(P)=\sum_{F} \mu(C(F, P)) \operatorname{vol}(F),
$$

where the sum is taken over all faces $F$ of $P$.

The existence of maps $\mu$ satisfying (2) was proved by McMullen McM], but his proof is not constructive. The difficulty is that (2) does not determine $\mu$ uniquely, so one must make some choices. The paper [BP] shows a way of unwinding McMullen's proof to yield what is in principle a constructive definition of a function $\mu$ satisfying (2), based on an initial choice of an ordered basis for the lattice containing $P$. However, computing their $\mu$ for any given cone is a rather complicated procedure.

We now describe our construction of a cycle Todd class in more detail. Let us suppose that we have a simplicial toric variety $X$ defined by a fan $\Sigma$ in a lattice $N$. We recall from Tho] a construction which shows that, having selected a complement map for $\Sigma$, we obtain a ring structure on $Z_{*}(X)_{\mathbb{Q}}$, the torus-invariant cycles of $X$ with rational coefficients. (We will define the notion of a complement map later; for now, let it suffice to say that choosing an inner product on $N \otimes \mathbb{Q}$ is one way to determine a complement map for any fan $\Sigma$ in $N$.)

$A_{*}(X)_{\mathbb{Q}}$, the Chow ring of cycles of $X$ modulo rational equivalence, is naturally a quotient of $Z_{*}(X)_{\mathbb{Q}}$; the ring structure on $Z_{*}(X)_{\mathbb{Q}}$ is defined so that this quotient map is a ring homomorphism.

There is a well-known expression for the Todd class of a smooth toric variety $X$ as a polynomial in the torus-invariant divisors, evaluated in $A_{*}(X)_{\mathbb{Q}}$. Once we have chosen a complement map for the lattice, we may evaluate this polynomial in $Z_{*}(X)_{\mathbb{Q}}$ instead and obtain a cycle that represents the Todd class of $X$. The formula for the dimension $k$ part of this cycle Todd class consists of a sum over cones of $\Sigma$ of codimension $k$ of a contribution which depends only on the cone itself, and not on data from other parts of the fan.

If our toric variety $X$ is not smooth, the standard way to compute the Todd class is to take a resolution of singularities $\pi: X^{\prime} \rightarrow X$, and then push forward the Todd class of $X^{\prime}$. We wish to mimic this process to construct a cycle Todd class for $X$. There is no problem taking a toric resolution of singularities, and the construction described above gives us a cycle representing the Todd class of $X^{\prime}$, which we can push forward to $X$. We denote this cycle by $t_{\Psi}^{X^{\prime}}(X)$, where $\Psi$ denotes the complement map.

The question that naturally arises is whether $t_{\Psi}^{X^{\prime}}(X)$ depends on $X^{\prime}$. Theorem 1 of this paper asserts that for a certain class of complement maps (called rigid complement maps, which include those induced from a choice of inner product), $t_{\Psi}^{X^{\prime}}(X)$ is independent of $X^{\prime}$. It follows that for any such $\Psi$, we have constructed an assignment of rational numbers to cones that satisfies Danilov's condition (Corollary $1)$.

Our proof of Theorem 1 relies on the formula of [Pom2] which expresses the Todd class of a complete, simplicial toric variety in a canonical way as a product of torus-invariant divisors with coefficients determined by the local combinatorics of the fan. Specifically, a function $f$ is constructed from tuples $\rho_{1}^{\left(a_{1}\right)}, \ldots, \rho_{r}^{\left(a_{r}\right)}$ of rays in a lattice $N$ with multiplicities $a_{i}>0$ to the rational numbers which satisfies

$$
\operatorname{Td}^{k}(X)=\sum f\left(\rho_{1}^{\left(a_{1}\right)}, \ldots, \rho_{r}^{\left(a_{r}\right)}\right)\left[V\left(\rho_{1}\right)\right]^{a_{1}} \cdots\left[V\left(\rho_{r}\right)\right]^{a_{r}}
$$


where the sum is taken of all tuples of rays in $\Sigma^{(1)}$ with $\sum a_{i}=k$. In [Pom2], $f$ is seen to satisfy reciprocity relations such as those satisfied by higher-dimensional Dedekind sums, and indeed the two-dimensional $f\left(\rho_{1}^{(1)}, \rho_{2}^{(1)}\right)$ is seen in Pom1] to be given by the classical Dedekind sum. If $X$ is complete and simplicial, then given a complement map for $X$, we obtain a cycle Todd class for $X$ by evaluating the formula of Pom2] in $Z_{*}(X)_{\mathbb{Q}}$. We denote this cycle Todd class by $t_{\Psi}^{s}(X)$.

The connection between these two approaches to cycle Todd classes is as follows: we show that if $X$ is complete and simplicial and $\Psi$ is rigid, then $t_{\Psi}^{s}(X)$ and $t_{\Psi}^{X^{\prime}}(X)$ coincide. It follows that for any $X, t_{\Psi}^{X^{\prime}}(X)$ does not depend on $X^{\prime}$.

One drawback to the cycle Todd classes described above is that they depend on a choice of complement map. We end the paper by discussing how to construct canonical cycle Todd classes, at the expense of expanding the coefficient field, as is done in Mor.

\section{The RING StruCture on $Z_{*}(X)_{\mathbb{Q}}$}

We begin by reviewing the results of [Tho]. Let $X$ be the toric variety determined by a fan $\Sigma$ in the lattice $N$. Let $Z_{i}(X)$ denote the free abelian group on the torus-invariant closed irreducible subvarieties of $X$ of dimension $i$. Let $Z_{*}(X)_{\mathbb{Q}}=$ $\bigoplus_{i=0}^{n} Z_{i}(X) \otimes \mathbb{Q}$. (A subscript $\mathbb{Q}$ will always indicate tensoring with $\mathbb{Q}$.)

For cones $\sigma, \tau$ in $N$, let us write $\tau \prec \sigma$ if $\tau$ is a face of $\sigma$. When we refer to cones, we always mean strictly convex rational polyhedral cones.

Definition. A complement map is a map $\Psi$ from a set of cones in $N$ to subspaces of $N$, satisfying the following two properties:

(i) $\Psi(\sigma)$ is complementary to the span of $\sigma$ in $N_{\mathbb{Q}}$.

(ii) If $\tau \prec \sigma$, and $\sigma$ is in the domain of $\Psi$, then $\tau$ is also in the domain of $\Psi$ and $\Psi(\sigma) \subset \Psi(\tau)$.

A complement map $\Psi$ is rigid if whenever $\sigma$ and $\tau$ are two cones with the same linear span such that $\Psi(\sigma)$ is defined, then $\Psi(\tau)$ is defined and equals $\Psi(\sigma)$.

There are two easy ways to construct complement maps. One way is to put an inner product on $N_{\mathbb{Q}}$ and let $\Psi(\sigma)$ be the orthogonal complement of the span of $\sigma$. Another way is to choose a flag $F_{0} \subset F_{1} \subset \cdots \subset F_{n}$ in $N_{\mathbb{Q}}$ and let $\Psi(\sigma)=F_{k}$ for all $\sigma$ of codimension $k$. This complement map can only be defined on those cones which intersect the fan $F_{*}$ generically. Observe that both these types of complement map are rigid.

The chief result of Tho is the following:

Proposition 1 (Tho]). If $\Sigma$ is a simplicial fan and $\Psi$ is a complement map defined on all the cones in $\Sigma$, then $\Psi$ induces a ring structure on $Z_{*}(X)_{\mathbb{Q}}$.

We wish to describe this ring structure briefly. To do this, we shall have to recall some basic notions about toric varieties. For further details, see [Ful].

Let $M$ be the dual lattice to $N$. For $\sigma$ a rational polyhedral cone in $N_{\mathbb{Q}}$, let $\widetilde{\sigma}$ denote the dual cone in $M_{\mathbb{Q}}: \check{\sigma}=\left\{x \in M_{\mathbb{Q}} \mid\langle x, y\rangle \geq 0\right.$ for all $\left.y \in \sigma\right\}$. Now $S_{\sigma}=M \cap \sigma$ is a semigroup, so we can form the semigroup ring $\mathbb{C}\left[S_{\sigma}\right]$, writing $\chi^{u}$ for the basis element of $\mathbb{C}\left[S_{\sigma}\right]$ corresponding to $u \in S_{\sigma}$. (The choice of base field is not important for our purposes; we use $\mathbb{C}$ for sake of definiteness.) Let $U_{\sigma}=\operatorname{Spec}\left(\mathbb{C}\left[S_{\sigma}\right]\right)$. 
Recall that $X$ has a covering by $U_{\sigma}$ for $\sigma \in \Sigma^{\max }$, the maximal cones in $\Sigma$. For the purposes of this exposition, we assume that all the maximal cones in $\Sigma$ are full-dimensional, though this is not in fact necessary. If $D$ is an equivariant Cartier divisor, it has, for each $\sigma \in \Sigma^{\max }$, a local equation on $U_{\sigma}$, which can be taken to be of the form $\chi^{m_{\sigma}}$ for some $m_{\sigma} \in M$. This collection of lattice points $m_{\sigma}$ will be referred to (somewhat abusively) as the local equations for $D$.

The closed subvariety $V(\tau)$ is defined by a fan in $N / \operatorname{span}(\tau)$, whose cones are $(\sigma+\operatorname{span}(\tau)) / \operatorname{span}(\tau)$ for $\sigma \succ \tau$. We can write $M_{\mathbb{Q}}=\tau^{\perp} \oplus \Psi(\tau)^{\perp}$, which induces a map $\pi_{\tau}: M_{\mathbb{Q}} \rightarrow \tau^{\perp}$. Given a Cartier divisor $D$, with local equations $m_{\sigma}$ for $\sigma \in \Sigma^{\max }$, define a Cartier divisor $D_{\tau}$ on $V(\tau)$ by taking its local equation on $(\sigma+\operatorname{span}(\tau)) / \operatorname{span}(\tau)$ to be $\pi_{\tau}\left(m_{\sigma}\right)$ for $\sigma \in \Sigma^{\max }$.

Now define $D \cdot V(\tau)=\left[D_{\tau}\right]$. Since any $[V(\sigma)]$ can be written as a product of $\mathbb{Q}$ Cartier divisors, this multiplication extends to define a multiplication on $Z_{*}(X)_{\mathbb{Q}}$. (Of course, one has to check that this multiplication is well-defined.)

In the case where the complement map $\Psi$ is induced from an inner product or a complete flag, we will give a presentation for $Z_{*}(X)_{\mathbb{Q}}$. For the case where $\Psi$ comes from an inner product, such a presentation was given in [Tho, which we restate as Proposition 2 below. For the case where $\Psi$ comes from a complete flag, we give a presentation for $Z_{*}(X)_{\mathbb{Q}}$ in Proposition 3 below, which is new.

Let $\Sigma$ be a simplicial fan, and let $\rho_{1}, \ldots, \rho_{r}$ be the rays of $\Sigma$, and let $D_{i}$ be the $\mathbb{Q}$-Cartier divisor corresponding to $V\left(\rho_{i}\right)$. Let $I_{\Sigma} \subset \mathbb{Q}\left[D_{1}, \ldots, D_{r}\right]$ denote the Stanley-Reisner ideal of the fan $\Sigma$, that is, the ideal generated by products of $D_{i}$ whose corresponding rays are not all contained in any cone of $\Sigma$. Let $v_{i}$ denote the first lattice point along $\rho_{i}$.

If the complement map is induced from an inner product, we have the following presentation for $Z_{*}(X)_{\mathbb{Q}}$ :

Proposition 2 ([Tho $]$ ). For $X$ a simplicial toric variety, if $\Psi$ is induced from an inner product, then

$$
Z_{*}(X)_{\mathbb{Q}} \cong \mathbb{Q}\left[D_{1}, \ldots, D_{r}\right] /\left(I_{\Sigma}+\left\langle D_{i} \sum_{1 \leq j \leq r} D_{j}\left\langle v_{i}, v_{j}\right\rangle \mid 1 \leq i \leq r\right\rangle\right) .
$$

We now provide a similar presentation for $Z_{*}(X)_{\mathbb{Q}}$ in the case where $\Psi$ is induced from a complete fan in $N_{\mathbb{Q}}$.

Let us write $\operatorname{div}\left(\chi^{u}\right)$ for the principal Cartier divisor whose local equation is $u$ on every $U_{\sigma}$. Fix a complete flag $F_{*}$ in $N_{\mathbb{Q}}$. Let $J$ denote the ideal of $\mathbb{Q}\left[D_{1}, \ldots, D_{r}\right]$ generated by terms of the form $\operatorname{div}\left(\chi^{u}\right) W$ where $W$ is homogeneous of degree $i$, and $u \in F_{n-i}^{\perp} \cap M$.

Proposition 3. For $X$ a simplicial toric variety, if $\Psi$ is induced from a complete flag $F_{*}$ in $N_{\mathbb{Q}}$, then

$$
Z_{*}(X)_{\mathbb{Q}} \cong \mathbb{Q}\left[D_{1}, \ldots, D_{r}\right] / I_{\Sigma}+J
$$

Proof. It is shown in [Tho that (no matter how $\Psi$ is chosen) there is a surjection from $\mathbb{Q}\left[D_{1}, \ldots, D_{r}\right]$ to $Z_{*}(X)_{\mathbb{Q}}$, whose kernel contains $I_{\Sigma}$.

Next, we show that if $\sigma$ is a cone of $\Sigma$ of codimension $i$, and $u \in F_{n-i}^{\perp} \cap M$, then $\operatorname{div}\left(\chi^{u}\right) \cdot[V(\sigma)]=0$. This follows by the definition of the multiplication given above: $\operatorname{div}\left(\chi^{u}\right)_{\sigma}$ is the divisor with all local equations zero. This shows that $Z_{*}(X)_{\mathbb{Q}}$ is a quotient of $\mathbb{Q}\left[D_{1}, \ldots, D_{r}\right] / I_{\Sigma}+J$.

To complete the proof, we will show that any term in $\mathbb{Q}\left[D_{1}, \ldots, D_{r}\right]$ is equivalent $\bmod I_{\Sigma}+J$ to a sum of squarefree monomials each of which is the product of 
divisors corresponding to rays which span a cone of $\Sigma$. This will show that, as abelian groups, $\mathbb{Q}\left[D_{1}, \ldots, D_{r}\right] / I_{\Sigma}+J \cong Z_{*}(X)_{\mathbb{Q}}$, which will prove the proposition.

Suppose that we have a monomial $Q$ in $D_{1}, \ldots, D_{r}$. For the sake of simplicity, suppose that $D_{1}$ occurs with multiplicity greater than 1 , and the $D_{i}$ which occur in $Q$ are $D_{1}, \ldots, D_{s}$. So let $Q=D_{1}^{a_{1}} \ldots D_{s}^{a_{s}}$. If $\rho_{1}, \ldots, \rho_{s}$ are not all contained in some cone of $\Sigma$, then $Q$ is already contained in $I_{\Sigma}$. So assume that they generate some cone $\sigma$. Then, by the genericity of $F_{*}, F_{n-s} \cap \operatorname{span}\left(\rho_{1}, \ldots, \rho_{s}\right)=\{0\}$. Thus, we can find $u \in F_{n-s}^{\perp} \cap M$, such that $\left\langle u, v_{1}\right\rangle \neq 0,\left\langle u, v_{i}\right\rangle=0$ for $2 \leq i \leq s$. Then $\operatorname{div}\left(\chi^{u}\right)$ expressed as a sum of $D_{i}$ has $D_{1}$ appearing with a non-zero coefficient, and possibly some $D_{i}$ with $i>s$. Now $\operatorname{div}\left(\chi^{u}\right) \cdot[V(\sigma)] \in J$. Thus, $\operatorname{div}\left(\chi^{u}\right) D_{1}^{a_{1}-1} D_{2}^{a_{2}} \ldots D_{s}^{a_{s}} \in J$. When we expand $\operatorname{div}\left(\chi^{u}\right) D_{1}^{a_{1}-1} D_{2}^{a_{2}} \ldots D_{s}^{a_{s}}$, we obtain a non-zero multiple of $Q$ plus terms which have strictly more distinct factors. Thus, mod $I_{\Sigma}+J, Q$ is equivalent to a sum of monomials which have strictly more distinct factors. Repeating this argument, we arrive eventually at a squarefree expression, which completes the proof that $Z_{*}(X)_{\mathbb{Q}} \cong \mathbb{Q}\left[D_{1}, \ldots, D_{r}\right] / I_{\Sigma}+J$.

We now recall a couple of basic notions of intersection theory as they apply to toric varieties. Let $X$ and $X^{\prime}$ be toric varieties defined by fans $\Sigma$ and $\Sigma^{\prime}$ in lattices $N$ and $N^{\prime}$. Let $f: X^{\prime} \rightarrow X$ be a torus-equivariant map, which implies that it is induced by a lattice map, also denoted $f$, from $N^{\prime}$ to $N$, such that for any $\sigma^{\prime} \in \Sigma^{\prime}$, $f\left(\sigma^{\prime}\right)$ is contained in some cone of $\Sigma$. Dualizing, $f$ also induces a map $f^{*}: M \rightarrow M^{\prime}$.

If $D$ is a Cartier divisor on $X$ with local equations $m_{\sigma}$, it can be pulled back in the usual way to $f^{*}(D)$ on $X^{\prime}$. The local equation of $f^{*}(D)$ on a cone $\sigma^{\prime} \in \Sigma^{\prime \max }$ is $f^{*}\left(m_{\sigma}\right)$, for $\sigma$ any cone of $\Sigma$ that contains $f\left(\sigma^{\prime}\right)$. This notion of pull-back clearly extends to $\mathbb{Q}$-Cartier divisors.

We shall also require the usual push-forward map of cycles: namely, for $f$ : $X^{\prime} \rightarrow X$ a morphism of algebraic varieties, $W^{\prime}$ a closed subvariety of $X^{\prime}$, and $W$ the Zariski closure of $f\left(W^{\prime}\right), f_{*}\left(\left[W^{\prime}\right]\right)=\left[k\left(W^{\prime}\right): f^{*}(k(W))\right][W]$, if $W$ is of the same dimension as $W^{\prime}$, and 0 otherwise. The push-forward map also has a natural description in terms of fans for torus-equivariant maps of toric varieties. This description is particularly simple in the case where $f: X^{\prime} \rightarrow X$ is also birational, which is the only case we shall need. The birationality of $f$ means that we can identify $N$ and $N^{\prime}$. Let $\sigma^{\prime} \in \Sigma^{\prime}$, and let $\sigma$ be the smallest cone of $\Sigma$ containing $\sigma^{\prime}$. Then $f_{*}\left(\left[V\left(\sigma^{\prime}\right)\right]\right)=[V(\sigma)]$ if $\sigma$ and $\sigma^{\prime}$ have the same dimension, and $f_{*}\left(\left[V\left(\sigma^{\prime}\right)\right]\right)=0$ otherwise.

We can now state a lemma which we shall need from Tho, a cycle level version of the projection formula from intersection theory.

Lemma 1 ([Tho]). Let $Y$ and $Z$ be simplicial toric varieties of the same dimension, $f: Y \rightarrow Z$ a proper torus-equivariant map, and $\Psi$ a rigid complement map for both $Y$ and $Z$. Let $D$ be $a \mathbb{Q}$-Cartier divisor on $Z, y \in Z_{*}(Y)_{\mathbb{Q}}$. Then

$$
f_{*}\left(f^{*}(D) \cdot y\right)=D \cdot f_{*}(y) .
$$

\section{A First APPROACH TO THE TOdD Class OF A TORIC VARIETY}

If $X$ is smooth, there is a formula for the Todd class of $X$, namely:

$$
\prod_{1 \leq i \leq r} \frac{D_{i}}{1-\exp \left(-D_{i}\right)}
$$


where the product is taken in $A_{*}(X)_{\mathbb{Q}}$. However, this product can just as well be interpreted in our ring $Z_{*}(X)_{\mathbb{Q}}$, and the result is a cycle which represents the Todd class. We denote this cycle by $t_{\Psi}(X)$.

Example 1. Let $\left\{e_{i}\right\}$ be the usual basis for $\mathbb{Z}^{n+1}$. Define a sublattice of $\mathbb{Z}^{n+1}$ by $N=\left\{\left(a_{1}, \ldots, a_{n+1}\right) \mid a_{i} \in \mathbb{Z}, \sum a_{i}=0\right\}$. Let $v_{i}=e_{i}-e_{i+1}$ and $v_{n+1}=e_{n+1}-e_{1}$. Let $\Sigma$ be the fan whose cones consist of positive linear combinations of proper subsets of the $v_{i}$. The toric variety associated to $\Sigma$ is $n$-dimensional projective space.

Now, put an inner product on $N$ by restriction of the usual inner product on $\mathbb{Z}^{n+1}$, i.e. set $\left\langle e_{i}, e_{j}\right\rangle=\delta_{i j}$. Let $\Psi$ be the complement map induced by this inner product. Then the coefficient of a cone $\sigma \in \Sigma$ in the cycle Todd class induced by $\Psi$ is the fraction of the linear span of $\sigma$ which is contained in $\sigma$. To prove this, one first reduces to the case where $\sigma$ is $n$-dimensional. By symmetry, the coefficients of all $n+1$ of the $n$-dimensional cones of $\Sigma$ must be the same. The sum of all the coefficients must be 1 , since the (Chow group) Todd class has $\operatorname{Td}^{n}\left(\mathbb{P}^{n}\right)=[p t]$. Thus the coefficients of each of the $n$-dimensional cones of $\Sigma$ are $\frac{1}{n+1}$, as desired. For further details of this calculation, see [Tho].

Smoothness for toric varieties has a simple interpretation in terms of the fan. We say that a cone is non-singular if the first lattice points along its extreme rays form a basis for the lattice contained in the linear span of the cone. Then a toric variety $X$ is smooth iff all the cones of its fan are non-singular.

Suppose $X$ is not smooth. Then the cones of $\Sigma$ can be subdivided, yielding a fan $\Sigma^{\prime}$, in such a way that all the cones of $\Sigma^{\prime}$ are non-singular. Let $X^{\prime}$ be the toric variety corresponding to $\Sigma^{\prime}$. Then $X^{\prime}$ is smooth, and there is a proper birational map from $X^{\prime}$ to $X$. The Todd class of $X$ coincides with the push-forward of the Todd class of $X^{\prime}$. Supposing that we have a complement map $\Psi$ for $X^{\prime}$, we can thus define a cycle Todd class for $X$, written $t_{\Psi}^{X^{\prime}}(X)$, as the pushforward of $t_{\Psi}\left(X^{\prime}\right)$.

A priori, this cycle Todd class $t_{\Psi}^{X^{\prime}}(X)$ depends on the choice of subdivision $\Sigma^{\prime}$ of $\Sigma$. We will show that under reasonable assumptions, $t_{\Psi}^{X^{\prime}}(X)$ does not depend on $\Sigma^{\prime}$. The chief result of this paper is the following theorem and its corollary:

Theorem 1. Let $X$ be any toric variety, with fan $\Sigma$. Let $\Psi$ be a rigid complement map and $X^{\prime}$ the toric variety induced by any non-singular subdivision $\Sigma^{\prime}$ of $\Sigma$ such that $\Psi$ is a complement map for $\Sigma^{\prime}$. Then the cycle Todd class $t_{\Psi}^{X^{\prime}}(X)$ does not depend on the choice of $X^{\prime}$.

Before giving the proof, we note that Theorem 1 allows us to construct from any rigid complement map a cycle Todd class with rational coefficients that satisfies Danilov's criterion.

Corollary 1. Fix a rigid complement map $\Psi$.

(i) Let $\sigma$ be a cone in the domain of $\Psi$. Then, for any fan $\Sigma$ containing $\sigma$, the coefficient of $[V(\sigma)]$ in the cycle Todd class of the toric variety defined by $\Sigma$ depends only on the cone $\sigma$ (and is otherwise independent of $\Sigma$ ). We call this coefficient the Todd measure of $\sigma$, and we denote it $\mu(\sigma)$.

(ii) (Danilov's question) If $\Psi$ is a rigid complement map defined on all cones in $N$, then the function $\mu$ obtained in this way satisfies Danilov's criterion: for any fan $\Sigma$ in $N$ with associated toric variety $X$, we have

$$
\operatorname{Td} X=\sum \mu(\sigma)[V(\sigma)] \text {. }
$$


(iii) (Additivity) If $\sigma_{1}, \ldots, \sigma_{t}$ are cones of the same dimension which overlap only on boundaries, such that their union is also a (strictly convex) cone $\tau$, and all the $\sigma_{i}$ and $\tau$ are in the domain of $\Psi$, then

$$
\mu(\tau)=\sum_{i=1}^{t} \mu\left(\sigma_{i}\right) .
$$

(Note that by the rigidity of $\Psi$, if any of the $\sigma_{i}$ or $\tau$ is in the domain of $\Psi$, so are all the others.)

(iv) Let $P$ be a lattice polytope in the dual lattice to $N, M=\operatorname{Hom}(N, \mathbb{Z})$. For $F$ a face of $P$, let $C(F, P)$ be the outer normal cone to $F$ in $N$. Let $\operatorname{vol}(F)$ denote the volume of $F$, normalized with respect to the lattice in the affine span of $F$.

Suppose that the outer normal fan of $P$ has a non-singular subdivision all of whose cones are in the domain of $\Psi$. Then we have the following formula for the number of lattice points of $P$ :

$$
\#(P)=\sum_{F} \mu(C(F, P)) \operatorname{vol}(F),
$$

where the sum is taken over all faces $F$ of $P$.

Proof. (i) We fix one way to refine $\sigma$ to a smooth fan, and then, using that refinement, we will always obtain the same coefficient of $[V(\sigma)]$ independent of $\Sigma$. By Theorem 1, this coefficient is also independent of the choice of refinement.

(ii) This follows immediately from the definition of $\mu$.

(iii) Let $\Sigma$ be a fan containing $\tau$ and $X$ the associated toric variety. Let $\Sigma^{\prime}$ be the fan obtained by replacing $\tau$ by $\sigma_{1}, \ldots, \sigma_{t}$ and $X^{\prime}$ the associated toric variety. Let $Y \rightarrow X^{\prime}$ be a resolution of singularities of $X^{\prime}$. The desired result follows from the fact that the coefficient of $[V(\tau)]$ in $t_{\Psi}^{Y}(X)$ is the sum of the coefficients of $\left[V\left(\sigma_{i}\right)\right]$ in $t_{\Psi}^{Y}\left(X^{\prime}\right)$.

(iv) This follows from (ii) by an application of Hirzebruch-Riemann-Roch; see Dan] or [Ful].

To prove Theorem 1, we shall connect the cycle Todd classes $t_{\Psi}^{X^{\prime}}(X)$ to the Todd class formula of [Pom2].

\section{A second approach to Cycle Todd Classes}

The Todd class formula from [Pom2] expresses the Todd class of a complete, simplicial toric variety in a canonical way as a product of Cartier divisors: for complete simplicial $X$, a polynomial $p_{X}$ is defined such that $p_{X}\left(D_{1}, \ldots, D_{r}\right)$, evaluated in the Chow ring with rational coefficients, gives the Todd class. Fix a complement map $\Psi$ for $X$. We define $t_{\Psi}^{s}(X)$ to be the result of evaluating $p_{X}\left(D_{1}, \ldots, D_{r}\right)$ in $Z_{*}(X)_{\mathbb{Q}}$. It follows that $t_{\Psi}^{s}(X)$ is a cycle Todd class of $X$.

We prove the following theorem:

Theorem 2. For $X$ a complete, simplicial toric variety, $X^{\prime} \rightarrow X$ a toric resolution of singularities of $X$, and $\Psi$ a rigid complement map for $X^{\prime}, t_{\Psi}^{s}(X)=t_{\Psi}^{X^{\prime}}(X)$. 
Proof. We begin by recalling the notion of a stellar subdivision of a fan. Let $\Sigma$ be a fan and $\rho_{0}$ a ray which is not a ray of $\Sigma$. The stellar subdivision of $\Sigma$ at $\rho_{0}$ consists of replacing every cone $\tau$ which contains $\rho_{0}$ by the cones $\rho_{0}+\delta$ where $\delta$ is a face of $\tau$ not containing $\rho_{0}$.

The main element in the proof of Theorem 2 is the following special case of the theorem:

Lemma 2. Let $\pi: Y \rightarrow Z$ be a proper birational map of simplicial toric varieties corresponding to a stellar subdivision. Then

$$
t_{\Psi}^{Y}(Z)=t_{\Psi}^{s}(Z) .
$$

Proof. Let $\rho_{0}$ be the ray that appears in the fan of $Y$ and not $Z$. Let $D_{0}$ be the $\mathbb{Q}$ Cartier divisor on $Y$ corresponding to $V\left(\rho_{0}\right)$. Let $\sigma$ be the minimal cone of the fan of $Z$ containing $\rho_{0}$. Let $E_{1}, \ldots, E_{d}$ be the $\mathbb{Q}$-Cartier divisors on $Z$ corresponding to rays in $\sigma$. Let $E_{d+1}, \ldots, E_{r}$ be the $\mathbb{Q}$-Cartier divisors on $Z$ corresponding to rays not in $\sigma$. For $1 \leq i \leq r$, let $D_{i}$ be the $\mathbb{Q}$-Cartier divisor on $Y$ corresponding to the same ray as $E_{i}$.

In [Pom2], a map $\tilde{\pi}$ from $\mathbb{Q}\left[y_{0}, \ldots, y_{r}\right]$ to $\mathbb{Q}\left[z_{1}, \ldots, z_{r}\right]$ is defined as follows:

(A) $\tilde{\pi}\left(y_{i} f\right)=z_{i} \tilde{\pi}(f)$ for $i>d$.

(B) $\tilde{\pi}\left(y_{i} f\right)=\frac{m_{i}}{m_{j}} \tilde{\pi}\left(y_{j} f\right)+\left(z_{i}-\frac{m_{i}}{m_{j}} z_{j}\right) \tilde{\pi}(f)$ for $1 \leq i, j \leq d$.

(C) $\tilde{\pi}\left(y_{0} f\right)=-\frac{m_{0}}{m_{j}} \tilde{\pi}\left(y_{j} f\right)+\frac{m_{0}}{m_{j}} z_{j} \tilde{\pi}(f)$ for $1 \leq j \leq d$.

(D) $\tilde{\pi}\left(y_{1} \ldots y_{d} f\right)=0$.

(E) $\tilde{\pi}\left(g\left(y_{1}, \ldots, y_{r}\right)\right)=g\left(z_{1}, \ldots, z_{r}\right)$ for $g$ any polynomial of degree at most $d-1$.

Here $m_{0}$ is the multiplicity of $\sigma$ (i.e. the index of the lattice generated by the first lattice points along the extreme rays of $\sigma$ in the intersection of $N$ with the span of $\sigma$ ), and $m_{i}$ is the multiplicity of the cone generated by the rays corresponding to $D_{0}, \ldots, \hat{D}_{i}, \ldots, D_{d}$.

It is relatively easy to show that there is at most one map $\tilde{\pi}$ with these properties. We will review the argument below, as we shall have need of it in a slightly different context. It is much harder to show that there is a map $\tilde{\pi}$ with all these properties. The reader is referred to [Pom2] for the proof of this.

By construction, $p_{Z}=\tilde{\pi}\left(p_{Y}\right)$. Therefore, we may apply the following lemma to show that $p_{Z}\left(E_{1}, \ldots, E_{r}\right)$ evaluated in $Z_{*}(Z)_{\mathbb{Q}}$ coincides with the pushforward of $p_{Y}\left(D_{0}, \ldots, D_{r}\right)$ evaluated in $Z_{*}(Y)_{\mathbb{Q}}$, which will prove Lemma 2 .

Lemma 3. Let $f$ be a polynomial in $y_{0}, \ldots, y_{r}$. Then we have the following equality in $Z_{*}(Z)_{\mathbb{Q}}$ :

$$
\tilde{\pi}(f)\left(E_{1}, \ldots, E_{r}\right)=\pi_{*}\left(f\left(D_{0}, \ldots, D_{r}\right)\right) .
$$

Proof. First, we check the following properties, where $W$ is any cycle in $Z_{*}(Y)$ and the $m_{i}$ are as above:

$\left(\mathrm{A}^{\prime}\right) \pi_{*}\left(D_{i} W\right)=E_{i}\left(\pi_{*}(W)\right)$ for $i>d$.

$\left(\mathrm{B}^{\prime}\right) \pi_{*}\left(D_{i} W\right)=\frac{m_{i}}{m_{j}} \pi_{*}\left(D_{j} W\right)+\left(E_{i}-\frac{m_{i}}{m_{j}} E_{j}\right) \pi_{*}(W)$ for $1 \leq i, j \leq d$.

$\left(\mathrm{C}^{\prime}\right) \pi_{*}\left(D_{0} W\right)=-\frac{m_{0}}{m_{j}} \pi_{*}\left(D_{j} W\right)+\frac{m_{0}}{m_{j}} E_{j} \pi_{*}(W)$ for $1 \leq j \leq d$.

$\left(\mathrm{D}^{\prime}\right) \pi_{*}\left(D_{1} \ldots D_{d} W\right)=0$.

(E') $\pi_{*}\left(g\left(D_{1}, \ldots, D_{r}\right)\right)=g\left(E_{1}, \ldots, E_{r}\right)$ for $g$ any polynomial of degree at most $d-1$. 
For $i>d, \pi^{*}\left(E_{i}\right)=D_{i}$. Thus, by an application of Lemma $1, \pi_{*}\left(D_{i} W\right)=$ $E_{i} \pi_{*}(W)$. This establishes $\left(\mathrm{A}^{\prime}\right)$. $\left(\mathrm{B}^{\prime}\right)$ and $\left(\mathrm{C}^{\prime}\right)$ follow similarly, using the facts that $\pi^{*}\left(m_{j} E_{i}-m_{i} E_{j}\right)=m_{j} D_{i}-m_{i} D_{j}$ and $\pi^{*}\left(m_{0} E_{j}\right)=m_{0} D_{j}+m_{j} D_{0} . \quad\left(\mathrm{D}^{\prime}\right)$ follows because $D_{1} \cdots D_{d}=0 \in Z_{*}(Y)_{\mathbb{Q}}$, since there are no cones containing all the corresponding rays. To establish $\left(\mathrm{E}^{\prime}\right)$, we observe that $g\left(D_{1}, \ldots, D_{r}\right)$ evaluated in $Z_{*}(Y)_{\mathbb{Q}}$ will consist of a sum of $V(\tau)$ with $\tau$ of codimension $d-1$ or less. For those $\tau$ not containing the ray corresponding to $D_{0}, V(\tau)$ will occur with the same coefficient in $g\left(D_{1}, \ldots, D_{r}\right)$ and in $g\left(E_{1}, \ldots, E_{r}\right)$. On the other hand, if $\tau$ does contain this ray, then $\pi_{*}([V(\tau)])=0$, because the image of $V(\tau)$ is $V(\sigma)$, which has lower dimension.

Next, we use essentially the argument of [Pom2] which establishes the uniqueness of $\tilde{\pi}$. Consider a monomial $Q=D_{0}^{a_{0}} \ldots D_{r}^{a_{r}}$. We wish to show that $\pi_{*}\left(D_{0}^{a_{0}} \ldots D_{r}^{a_{r}}\right)$ is determined by properties $\left(\mathrm{A}^{\prime}\right)$ through $\left(\mathrm{E}^{\prime}\right)$. The proof is inductive. ( $\left.\mathrm{E}^{\prime}\right)$ establishes the result for terms of degree at most $d-1$. Now assume the result for terms of lower degree than $Q$. If any $a_{i}$ are greater than zero for $i>d$, apply $\left(\mathrm{A}^{\prime}\right)$, and the desired result follows by induction. If there is some $1 \leq j \leq d$ such that $a_{j}$ is zero, apply either $\left(\mathrm{B}^{\prime}\right)$ or $\left(\mathrm{C}^{\prime}\right)$ to replace $Q$ by terms which can be dealt with by induction, plus a term which has fewer $i, 1 \leq i \leq d$, such that $a_{i}=0$. Repeat this process until the problematic term has $a_{i} \neq 0$ for $1 \leq i \leq d$, at which point it can be disposed of by $\left(\mathrm{D}^{\prime}\right)$.

Now observe that, by properties (A) through (E), the map taking a polynomial $f \in \mathbb{Q}\left[y_{0}, \ldots, y_{r}\right]$ to $\tilde{\pi}(f)\left(E_{1}, \ldots, E_{r}\right) \in Z_{*}(Z)_{\mathbb{Q}}$ has properties $\left(\mathrm{A}^{\prime}\right)$ through $\left(\mathrm{E}^{\prime}\right)$, and thus it coincides with the map taking $f$ to $\pi_{*}\left(f\left(D_{0}, \ldots, D_{r}\right)\right)$, as desired.

This establishes Lemma 3. As already described, Lemma 2 follows.

Now, we turn to the proof of Theorem 2. Let $W=W_{p} \rightarrow W_{p-1} \rightarrow \cdots \rightarrow$ $W_{0}=X$ be a resolution of singularities for $X$ such that the map from $W_{i}$ to $W_{i-1}$ is induced by a stellar subdivision of fans. (The existence of a resolution of singularities of this type for any $X$ is shown in Ful].) Let $w_{i}$ denote the map from $W_{i}$ to $X$.

Now, we prove by induction on $i$ that $\left(w_{i}\right)_{*}\left(t_{\Psi}^{s}\left(W_{i}\right)\right)=t_{\Psi}^{s}(X)$. The $i=0$ case is trivial. The induction step consists in observing that $w_{i+1}$ factors through $W_{i}$, and then applying Lemma 2. This establishes that $t_{\Psi}^{W}(X)=t_{\Psi}^{s}(X)$.

We must now prove that $t_{\Psi}^{W}(X)=t_{\Psi}^{X^{\prime}}(X)$ for $X^{\prime} \rightarrow X$ any resolution of singularities. We recall the Weak Oda Theorem:

Weak Oda Theorem ( $(\overline{W l}])$. Given $\Pi$ and $\Sigma$ two non-singular fans with the same support, there is a sequence of non-singular fans $\Pi=\Delta_{0}, \Delta_{1}, \ldots, \Delta_{p}=\Sigma$ such that either $\Delta_{i+1}$ is obtained from $\Delta_{i}$ by a stellar subdivision or vice versa.

Further, if both $\Pi$ and $\Sigma$ refine some fan $\Omega$, then all the $\Delta_{i}$ may be chosen so as to refine $\Omega$.

Applying the Weak Oda Theorem to $W$ and $X^{\prime}$ (both of whose fans refine the fan for $X$ ), we obtain a sequence of toric varieties $X^{\prime}=Z_{p}, \ldots, Z_{0}=W$, all of whose fans refine the fan of $X$, such that either $Z_{i+1}$ is obtained from $Z_{i}$ by a stellar subdivision or vice versa. Lemma 2 implies that $t_{\Psi}^{W_{i}}(X)=t_{\Psi}^{W_{i+1}}(X)$, and it follows that $t_{\Psi}^{X^{\prime}}(X)=t_{\Psi}^{W}(X)$, as desired.

We now prove Theorem 1. 
Proof. If $X$ is complete and simplicial, then by Theorem $2, t_{\Psi}^{X^{\prime}}(X)=t_{\Psi}^{s}(X)$, so $t_{\Psi}^{X^{\prime}}(X)$ does not depend on $X^{\prime}$. However, we must establish the result without the assumption that $X$ is complete and simplicial.

Suppose first that $X$ is complete but not simplicial. Let $Y \rightarrow X$ and $Y^{\prime} \rightarrow X$ be two resolutions of singularities. We can always construct a resolution of singularities $Z \rightarrow X$ which factors through both $Y$ and $Y^{\prime}$ by taking a fan which is a subdivision of both $Y$ and $Y^{\prime}$ and then subdividing it further so as to make it non-singular. Thus, it suffices to show that if $g: Z \rightarrow Y$ and $f: Y \rightarrow X$, such that $f$ and $f \circ g$ are resolutions of singularities for $X$, then $t_{\Psi}^{Y}(X)=t_{\Psi}^{Z}(X)$. Now

$$
\begin{aligned}
t_{\Psi}^{Z}(X) & =(f \circ g)_{*}\left(t_{\Psi}(Z)\right) \\
& =f_{*}\left(g_{*}\left(t_{\Psi}(Z)\right)\right) \\
& =f_{*}\left(t_{\Psi}(Y)\right) \\
& =t_{\Psi}^{Y}(X)
\end{aligned}
$$

where we have used the fact that $g_{*}\left(t_{\Psi}(Z)\right)=t_{\Psi}(Y)$, by Theorem 2 .

Now suppose that $X$ is not complete. Let $\tilde{X}$ be a toric variety obtained by adding cones to the fan $\Sigma$ for $X$, in order to make it complete. As before, let $Y \rightarrow X$ and $Y^{\prime} \rightarrow X$ be resolutions of singularities, and let $\tilde{Y}$ and $\tilde{Y}^{\prime}$ be resolutions of singularities for $\tilde{X}$ whose fans intersected with the support of $\Sigma$ agree with those of $Y$ and $Y^{\prime}$, respectively. For any $\sigma \in \Sigma$, the coefficient of $[V(\sigma)]$ in $t_{\Psi}^{Y}(X)$ is the same as the coefficient of $t_{\Psi}^{\tilde{Y}}(\tilde{X})$, and similarly replacing $Y$ by $Y^{\prime}$ and $\tilde{Y}$ by $\tilde{Y}^{\prime}$. Thus, the general result follows by applying the result in the complete case to $\tilde{X}$. This proves Theorem 1 .

\section{Cycle Todd Classes induced By A ChOice of FlaG}

In this section we investigate further $t_{\Psi}(X)$ for $X$ smooth and $\Psi$ induced by a complete flag. Our tool will be a more general result about computation in $Z_{*}(X)_{\mathbb{Q}}$ when $\Psi$ is induced from a complete flag.

Theorem 3. Let $X$ be a simplicial toric variety, and let $\Psi$ be induced from a complete flag $F_{*}$. Let $k \leq n$, and let $a_{1}, \ldots, a_{k}$ be non-negative integers that sum to $k$. Let $\sigma$ be the cone spanned by $\rho_{1}, \ldots, \rho_{k}$. Let $v_{i}$ be the first lattice point along $\rho_{i}$. By the genericity of $F_{*}$, there is a unique (up to scalar multiple) collection of non-zero $t_{i}$ such that

$$
\sum_{i=1}^{k} t_{i} v_{i} \in F_{n-k+1} .
$$

Then the coefficient of $[V(\sigma)]$ in $\prod_{i=1}^{k} D_{i}^{a_{i}}$ is

$$
\frac{1}{\operatorname{mult} \sigma} \prod_{i=1}^{k} t_{i}^{a_{i}-1} \text {. }
$$

Proof. Most of our effort will be directed to showing that the coefficient of $[V(\sigma)]$ in $\prod_{i=1}^{k} D_{i}^{a_{i}}$ coincides with the coefficient of $[V(\sigma)]$ in $\prod_{i=1}^{k} t_{i}^{a_{i}-1} D_{i}$. The proof is by induction on the number of $a_{i}$ which equal zero. The base case, when none of the $a_{i}=0$, and thus all the $a_{i}=1$, is tautological.

So suppose there is some $a_{l}=0$. Pick $j$ such that $a_{j}>1$. Observe that there is an element $m \in F_{n-k+1}^{\perp}$ such that $\left\langle m, v_{j}\right\rangle=t_{l},\left\langle m, v_{l}\right\rangle=-t_{j}$, and $\left\langle m, v_{i}\right\rangle=0$ for 
all $i \neq j, l$. By Proposition 3, for any $\tau \prec \sigma \in \Sigma$ of dimension $k-1$,

$$
\operatorname{div}\left(\chi^{m}\right) \cdot[V(\tau)]=0
$$

and thus the coefficients of $[V(\sigma)]$ in $t_{l} D_{j} \cdot[V(\tau)]$ and $t_{j} D_{l} \cdot[V(\tau)]$ coincide.

Consequently, the coefficient of $[V(\sigma)]$ in $D_{1}^{a_{1}} \cdots D_{k}^{a_{k}}$ is the same as the coefficient of $[V(\sigma)]$ in

$$
\frac{t_{j}}{t_{l}} D_{1}^{a_{1}} \cdots D_{j}^{a_{j}-1} \cdots D_{l}^{a_{l}+1} \cdots D_{k}^{a_{k}}
$$

This expression has one fewer $a_{i}$ which is zero, and our claim follows by induction.

The statement of the corollary now follows from the fact that

$$
D_{1} \cdots D_{k}=\frac{1}{\operatorname{mult} \sigma}[V(\sigma)]
$$

The following corollary states that for a complement map induced by a complete flag, the Todd class $t_{\Psi}(X)$ we have constructed coincides with the Todd class of [Mor, Theorem 11]. For each $k$-dimensional cone $\sigma$, Morelli defines $\mu_{k}^{t d_{k}}(\sigma)$, a rational function on the Grassmannian $G_{n-k+1}(N)$ of $n-k+1$-planes in $N$. In our $t_{\Psi}(X)$, the coefficient $\mu(\sigma)$ of the torus-invariant cycle $[V(\sigma)]$ depends only on $F_{n-k+1}$, where $k$ is the dimension of $\sigma$, and not on the rest of the flag. In this way we can view $\mu$ as a rational function on $G r_{n-k+1}(N)$. The corollary below assures us that this function is exactly $\mu_{k}^{t d_{k}}(\sigma)$.

Corollary 2. Let $\mu_{k}^{t d_{k}}$ denote the function of $\underline{\text { Mor, }}$, Theorem 11]. Let $F$ be a complete flag, let $\Psi$ be the induced complement map, and let $\mu$ be the associated Todd measure (as in Corollary 1(i)). Then for any cone of dimension $k$,

$$
\mu(\sigma)=\mu_{k}^{t d_{k}}(\sigma)\left(F_{n-k+1}\right) .
$$

Proof. For non-singular $\sigma$, the equation follows by comparing Theorem 3 to the definition of $\mu_{k}^{t d_{k}}$ [Mor, p. 218].

Suppose that $\sigma$ is singular. Let $\Sigma$ be a fan consisting of $\sigma$ and its faces, and let $\Sigma^{\prime}$ be a desingularization of $\Sigma$. Let $\tau_{1}, \ldots, \tau_{m}$ be the $k$-dimensional cones of $\Sigma^{\prime}$. By the additivity of $\mu_{k}^{t d_{k}}$ [Mor, p. 184], $\mu_{k}^{t d_{k}}(\sigma)=\sum_{i=1}^{m} \mu_{k}^{t d_{k}}\left(\tau_{i}\right)$. By the additivity of $\mu$ (Corollary 1(iii)), the same formula holds with $\mu$ replacing $\mu_{k}^{t d_{k}}$ everywhere, which reduces us to the non-singular case.

\section{Canonical cycle Todd classes}

A drawback of the cycle Todd classes presented so far is that they depend on a choice of complement map. Further, no single choice of fan defines a complement map simultaneously for all cones in $N$. Both these problems can be avoided, as is done in Mor], by enlarging the coefficient field of the cycles. In this way we obtain a canonical Todd class based on the inner product construction and one based on the complete flag construction.

Let $\mathcal{F}$ be the set of all complete flags in $N_{\mathbb{Q}}$, which has the structure of an algebraic variety. For $F$ a complete flag in $N_{\mathbb{Q}}$, generic with respect to the fan for $X$, let $t_{F}(X)$ be the cycle Todd class for $X$ given by the complement map induced by $F$. Now, define $t_{\mathcal{F}}(X): \mathcal{F} \rightarrow Z_{*}(X)_{\mathbb{Q}}$ by $t_{\mathcal{F}}(X)(F)=t_{F}(X)$ when $t_{F}(X)$ is defined. We observe that, by the results of the previous section, $t_{\mathcal{F}}(X)$ is a rational 
function on $\mathcal{F}$, and thus if we write $\mathbb{K}$ for the rational function field of $\mathcal{F}$, we can view $t_{\mathcal{F}}(X)$ as lying in $Z_{*}(X)_{\mathbb{K}}$, and it is clear that it is a cycle Todd class for $X$.

One can do essentially the same thing to produce a canonical Todd class based on the inner product construction. We construct Todd classes in the rational function field of $\mathcal{S}$, the set of all symmetric $n \times n$ matrices. Fixing a basis for $N$, we can identify inner products on $N_{\mathbb{Q}}$ with symmetric positive definite $n \times n$ matrices. Using the approach of the previous paragraph, we define cycle Todd classes whose coefficients are functions from symmetric positive definite $n \times n$ matrices to $\mathbb{Q}$; one then checks that these functions extend to rational functions on $\mathcal{S}$.

\section{ACKNOWLEDGEMENTS}

The authors thank William Fulton and Burt Totaro for much useful advice, and they thank the referee for many helpful suggestions, including an improvement to the statement of Theorem 3 .

\section{REFERENCES}

[BP] A. Barvinok and J. E. Pommersheim, An algorithmic theory of lattice points in polyhedra, New Perspectives in Algebraic Combinatorics, MSRI Publications 38, 1999, pp. 91-147. MR1731815 (2000k:52014)

[Dan] V. I. Danilov, The geometry of toric varieties, Russian Math. Surveys 33:2 (1978), 97-154. MR0495499 (80g:14001)

[Ful] W. Fulton, Introduction to Toric Varieties, Annals of Mathematics Studies Number 131, Princeton University Press, Princeton, 1993. MR.1234037|(94g:14028)

$[\mathrm{McM}]$ P. McMullen, Weakly continuous valuations on convex polytopes, Archiv Math. 41 (1983) 555-564. MR0731639 (85i:52002)

[Mor] R. Morelli, Pick's theorem and the Todd class of a toric variety, Adv. Math. 100:2 (1993), 183-231. MR1234309 (94j:14048)

[Pom1] J. E. Pommersheim, Toric varieties, lattice points and Dedekind sums, Math. Ann. 295 (1993) 1-24. MR1198839 (94c:14043)

[Pom2] J. E. Pommersheim, Products of Cycles and the Todd Class of a toric variety, J. Amer. Math. Soc. 9 (1996) 813-826. MR.1358042 (96j:14037)

[Tho] H. Thomas, Cycle-level intersection theory for toric varieties, to appear in Canad. J. Math., available at http://www.arxiv.org/math.AG/0306144.

[Wlo] J. Włodarczyk, Decomposition of birational toric maps in blow-ups and blow-downs, Trans. Amer. Math. Soc. 349 (1997), 373-411. MR1370654(97d:14021)

Department of Mathematics, Pomona College, Claremont, California 92037

E-mail address: jpommersheim@pomona.edu

Fields Institute, 222 College Street, Toronto ON, M5T 3J1 Canada

E-mail address: hthomas@fields.utoronto.ca

Current address: Department of Mathematics and Statistics, University of New Brunswick, Fredericton, New Brunswick, E3B 5A3 Canada 